\title{
A multi-institutional analysis of the untreated course of cerebral dural arteriovenous fistulas
}

\author{
Bradley A. Gross, MD, ${ }^{1,2}$ Felipe C. Albuquerque, MD, ${ }^{4}$ Cameron G. McDougall, MD, ${ }^{5}$ \\ Brian T. Jankowitz, MD, ${ }^{1,2}$ Ashutosh P. Jadhav, MD, PhD, ${ }^{1,3}$ Tudor G. Jovin, MD, ${ }^{1,3}$ and \\ Rose Du, MD, PhD ${ }^{6}$
}

\begin{abstract}
1UPMC Stroke Institute, Departments of ${ }^{2}$ Neurosurgery and ${ }^{3}$ Neurology, University of Pittsburgh Medical Center, Pittsburgh, Pennsylvania; ${ }^{4}$ Department of Neurosurgery, Barrow Neurological Institute, St. Joseph's Hospital and Medical Center, Phoenix, Arizona; ${ }^{5}$ Swedish Cerebrovascular Center, Swedish Neuroscience Institute, Seattle, Washington; and ${ }^{6}$ Department of Neurological Surgery, Brigham and Women's Hospital and Harvard Medical School, Boston, Massachusetts
\end{abstract}

OBJECTIVE The rarity of cerebral dural arteriovenous fistulas (dAVFs) has precluded analysis of their natural history across large cohorts. Investigators from a considerable proportion of the few reports that do exist have evaluated heterogeneous groups of untreated and partially treated lesions. In the present study, the authors exclusively evaluated the untreated course of dAVFs across a multi-institutional data set to delineate demographic, angiographic, and natural history data.

METHODS A multi-institutional database of dAVFs was queried for demographic and angiographic data as well as untreated disease course. After dAVFs were stratified by Djindjian type, annual nonhemorrhagic neurological deficit (NHND) and hemorrhage rates were derived, as were risk factors for each. A multivariable Cox proportional-hazards regression model was used to calculate hazard ratios.

RESULTS Two hundred ninety-five dAVFs had at least 1 month of untreated follow-up. For 126 Type I dAVFs, there were no episodes of NHND or hemorrhage over 177 lesion-years. Respective annualized NHND and hemorrhage rates were $4.5 \%$ and $3.4 \%$ for Type II, $6.0 \%$ and $4.0 \%$ for Type III, and $4.5 \%$ and $9.1 \%$ for Type IV dAVFs. The respective annualized NHND and hemorrhage rates were $2.3 \%$ and $2.9 \%$ for asymptomatic Type II-IV dAVFs, $23.1 \%$ and $3.3 \%$ for dAVFs presenting with NHND, and $0 \%$ and $46.2 \%$ for lesions presenting with hemorrhage. On multivariate analysis, NHND presentation (HR 11.49, 95\% Cl 3.19-63) and leptomeningeal venous drainage (HR 5.03, 95\% Cl 0.42-694) were significant risk factors for NHND; hemorrhagic presentation (HR 17.67, 95\% Cl 2.99-117) and leptomeningeal venous drainage (HR 10.39, 95\% Cl 1.11-1384) were significant risk factors for hemorrhage.

CONCLUSIONS All Type II-IV dAVFs should be considered for treatment. Given the high risk of rebleeding, lesions presenting with NHND and/or hemorrhage should be treated expediently.

https://thejns.org/doi/abs/10.3171/2017.6.JNS171090

KEY WORDS dural arteriovenous fistula; dAVF; epidemiology; hemorrhage; cortical venous drainage; natural history; vascular disorders

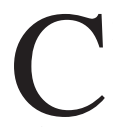

EREBRAL dural arteriovenous fistulas (dAVFs) are vascular shunts that are unique to the neuraxis as the shunt site is contained within the dural leaflets. dAVFs are supplied by meningeal arteries with drainage into a venous sinus or leptomeningeal veins. Decades ago, drainage or reflux into leptomeningeal veins was identified as the cardinal angiographic feature that can portend a more aggressive course as a result of intracranial venous hypertension. ${ }^{7}$ Resultant neurological deficits can masquerade as ischemic infarction, parkinsonism, or even dementia. ${ }^{8-10}$ Resultant hemorrhage is associated with considerable neurological morbidity and mortality. ${ }^{3,6,12-15}$

A few single-center series have provided insight into the importance of venous drainage and presentation modality as risk factors for nonhemorrhagic neurological deficits (NHNDs; including ischemic symptoms, demen-

ABBREVIATIONS dAVF = dural arteriovenous fistula; NHND = nonhemorrhagic neurological deficit.

SUBMITTED May 1, 2017. ACCEPTED June 5, 2017.

INCLUDE WHEN CITING Published online December 15, 2017; DOI: 10.3171/2017.6.JNS171090. 
tia, parkinsonism, seizures) and/or hemorrhage..$^{3,12-15} \mathrm{We}$ previously pooled these results to demonstrate a statistically significant impact of presentation modality and venous ectasia on the risk of NHND and/or hemorrhage. ${ }^{6}$ Nevertheless, these studies were limited by small sample sizes and the inclusion of a considerable proportion of partially treated dAVFs. ${ }^{3,12-15}$ In the present analysis, we exclusively evaluated the untreated course of dAVFs across a multi-institutional data set to delineate demographic, angiographic, and natural history data.

\section{Methods}

Cerebrovascular databases at 3 institutions (Barrow Neurological Institute, Brigham and Women's Hospital, and University of Pittsburgh) were queried for patients with cerebral dAVFs. Where applicable, databases were maintained in accordance with local institutional review committees. The records of patients with at least 1 month of untreated follow-up were reviewed. Patient age at presentation, sex, presentation modality, fistula location, fistula drainage pattern, and follow-up period were extracted from the records. Dural AVFs were classified based on the original Djindjian classification scheme. ${ }^{4}$ Type I dAVFs drain into a venous sinus, Type II dAVFs drain into a venous sinus with leptomeningeal venous reflux, Type III dAVFs drain directly into leptomeningeal veins, and Type IV dAVFs drain into leptomeningeal veins with ectasia. "Patient-years of follow-up" was defined as the number of years from the time of presentation until the last clinical follow-up or the initiation of any treatment modality (embolization, surgery, or radiosurgery). During this followup period, the timing of any new NHND or hemorrhagic event was noted by the treating or monitoring surgeon.

\section{Statistical Analysis}

The Cox proportional hazards regression model with Firth's penalized maximum likelihood bias reduction method was used for the survival analyses, which were performed with the "coxphf" package in R (version 3.3.2, $\mathrm{R}$ Foundation for Statistical Computing). Evaluated censoring events included hemorrhage and NHND. "Initial time" was considered to be the time of initial clinical presentation in symptomatic cases and the time of initial imaging diagnosis for incidental cases. Univariate and multivariate analyses were performed for hemorrhage presentation, NHND presentation, patient age, female sex, leptomeningeal venous drainage, and venous ectasia.

\section{Results}

Across the 3 institutions, 276 patients with 295 dAVFs had at least 1 month of untreated follow-up and thus were included in our analysis with a total of 384 lesion-years. Mean patient age was $58.7 \pm 15.3$ years (mean \pm standard deviation), and $55 \%$ of the patients were male (1.2:1 male/female). The most common dAVF location was the transverse/sigmoid sinus (33\% of cases). Remaining locations included the cavernous sinus (19\%), tentorial/petrosal sinus (18\%), superior sagittal sinus/convexity (12\%), marginal sinus/jugular bulb (7\%), torcula (5\%), ethmoidal/ anterior fossa (4\%), and sphenoparietal sinus (1\%). Categorizing by Djindjian type, $43 \%$ were Type I, $22 \%$ were Type II, $25 \%$ were Type III, and $10 \%$ were Type IV. Sixteen percent of the dAVFs were incidental/asymptomatic. Forty percent were associated with pulsatile tinnitus and $20 \%$ with ocular symptoms (chemosis and/or proptosis and/or diplopia). Nine percent of the dAVFs were hemorrhagic on presentation, and $13 \%$ were associated with NHNDs. Demographics, presentations, and dAVF locations are stratified by Djindjian type in Table 1.

\section{Low-Risk dAVFs: Type I}

Our combined cohort comprised 126 dAVFs without leptomeningeal venous drainage (Type I). Mean patient age was 53.6 years, and there was no sex predilection (1:1 male/female). The most common dAVF location was the transverse/sigmoid sinus (44\%). Other locations included the cavernous sinus (32\%), marginal sinus $(14 \%)$, torcula $(5 \%)$, superior sagittal sinus $(4 \%)$, tentorial/petrosal sinus $(1 \%)$, and ethmoidal $(1 \%)$. No patients presented with NHND or hemorrhage, and none went on to develop NHNDs or hemorrhage over a total of 177 lesion-years (mean 1.4 lesion-years). Four dAVFs went on to spontaneously thrombose (3\%).

\section{High-Risk dAVFs: Types II-IV}

One hundred sixty-nine dAVFs were associated with leptomeningeal venous reflux. Stratified by Djindjian classification, all high-risk types had a male sex predilection, particularly Type IV dAVFs (72\% male; Table 1). Locations for Type II dAVFs were generally similar to those for Type I dAVFs: $56 \%$ were transverse/sigmoid and $24 \%$ were cavernous. Types III and IV dAVFs had similar proportions of tentorial/petrosal (50\% and 51\%, respectively) and superior sagittal sinus/convexity dAVFs (28\% and $24 \%$, respectively). A considerable proportion of Type IV dAVFs were ethmoidal (24\%). Evaluating the venous drainage pattern for each dAVF location, we found the

TABLE 1. Demographics, presentation, and fistula location, stratified by Djindjian type

\begin{tabular}{lcccc}
\hline \multicolumn{1}{c}{ Parameter } & Type I & Type II & Type III & Type IV \\
\hline No. of dAVFs & 126 & 66 & 74 & 29 \\
\hline Mean age in yrs & 53.6 & 57.5 & 60.7 & 60.0 \\
\hline Male (\%) & 50 & 55 & 59 & 72 \\
\hline Aggressive presentation (\%) & & & & \\
$\quad$ NHND & 0 & 18 & 24 & 31 \\
Hemorrhage & 0 & 6 & 22 & 28 \\
\hline Location (\%) & & & & \\
Transverse/sigmoid sinus & 44 & 56 & 5 & 0 \\
Cavernous sinus & 32 & 24 & 0 & 0 \\
Marginal sinus & 14 & 5 & 1 & 0 \\
Torcula & 5 & 12 & 3 & 0 \\
Tentorial/petrosal sinus & 1 & 0 & 50 & 51 \\
Superior sagittal sinus/convexity & 4 & 3 & 28 & 24 \\
Sphenoparietal sinus & 0 & 0 & 5 & 0 \\
Ethmoidal & 1 & 0 & 7 & 24 \\
\hline
\end{tabular}


TABLE 2. Stratification of venous drainage patterns for each dAVF location

\begin{tabular}{|c|c|c|c|c|c|}
\hline Location & $\begin{array}{l}\text { No. of } \\
\text { dAVFs }\end{array}$ & $\begin{array}{c}\text { Type } \\
\text { । } \\
(\%)\end{array}$ & $\begin{array}{c}\text { Type } \\
\text { II } \\
(\%)\end{array}$ & $\begin{array}{c}\text { Type } \\
\text { III } \\
(\%)\end{array}$ & $\begin{array}{c}\text { Type } \\
\text { IV } \\
(\%)\end{array}$ \\
\hline Transverse/sigmoid sinus & 96 & 58 & 39 & 4 & 0 \\
\hline Cavernous sinus & 56 & 71 & 29 & 0 & 0 \\
\hline Marginal sinus & 22 & 81 & 14 & 5 & 0 \\
\hline Torcula & 16 & 38 & 50 & 13 & 0 \\
\hline Tentorial/petrosal sinus & 53 & 2 & 0 & 70 & 28 \\
\hline Superior sagittal sinus/convexity & 35 & 14 & 6 & 60 & 20 \\
\hline Sphenoparietal sinus & 4 & 0 & 0 & 100 & 0 \\
\hline Ethmoidal & 13 & 8 & 0 & 38 & 54 \\
\hline
\end{tabular}

majority of sphenoparietal sinus (100\%), tentorial/petrosal (98\%), ethmoidal (92\%), and superior sagittal sinus/ convexity $(86 \%)$ were high risk (Types II-IV; Table 2). Ethmoidal dAVFs had the highest proportion of Type IV lesions (54\%). Torcular dAVFs had a greater tendency to harbor leptomeningeal venous reflux than the transverse/ sigmoid dAVFs (63\% vs 43\%, respectively). Marginal si- nus and cavernous sinus dAVFs had the lowest proportion of Type II-IV lesions (19\% and 29\%, respectively).

\section{Hemorrhage and NHND Rates}

Respective annualized NHND and hemorrhage rates were $4.5 \%$ and $3.4 \%$ for Type II, $6.0 \%$ and $4.0 \%$ for Type III, and $4.5 \%$ and $9.1 \%$ for Type IV dAVFs (Fig. 1). There was a total of 115 lesion-years of follow-up for Type II dAVFs (mean 1.6 lesion-years), 69 lesion-years for Type III (mean 0.9 lesion-years), and 23 lesion-years for Type IV (mean 0.8 lesion-years). Annualized NHND and hemorrhage rates were $2.3 \%$ and $2.9 \%$ for asymptomatic Type II-IV dAVFs, $23.1 \%$ and $3.3 \%$ for dAVFs presenting with NHND, and $0 \%$ and $46.2 \%$ for dAVFs presenting with hemorrhage, respectively. There was a total of 125 lesionyears of follow-up for asymptomatic Type II-IV dAVFs (mean 1.2 lesion-years), 74 lesion-years of follow-up for dAVFs presenting with NHND (mean 1.9 lesion-years), and 8 lesion-years of follow-up for dAVFs presenting with hemorrhage (mean 0.3 lesion-years).

In our univariate survival analysis, older age (HR 1.07, 95\% CI 1.03-1.13), NHND presentation (HR 32.91, 95\% CI 9.40-173), leptomeningeal venous drainage (HR 23.66, 95\% CI 3.14-3029), and venous ectasia (HR 4.21, 95\%

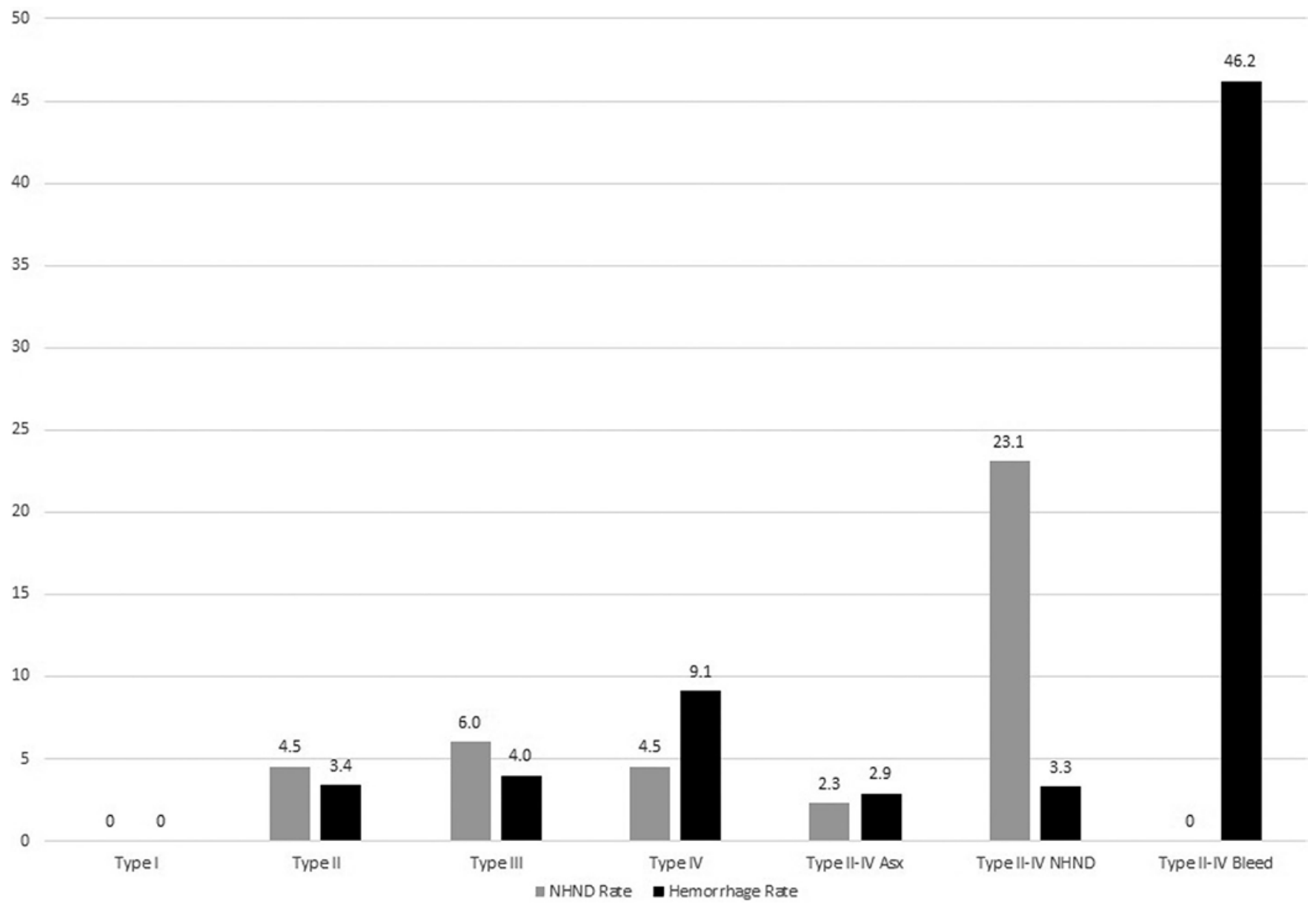

FIG. 1. Annual NHND and hemorrhage rates stratified by Djindjian type and presentation modality. Asx = asymptomatic, but also refers to any patient who did not present with either an NHND or a hemorrhage. 
TABLE 3. Evaluation of risk factors for the development of new NHNDs and hemorrhage

\begin{tabular}{lccccc}
\hline \multirow{2}{*}{ Factor } & \multicolumn{2}{c}{ NHND } & & \multicolumn{2}{c}{ Hemorrhage } \\
\cline { 2 - 3 } \cline { 6 - 7 } & $\begin{array}{c}\text { Univariate HR } \\
(95 \% \mathrm{Cl})\end{array}$ & $\begin{array}{c}\text { Multivariate HR } \\
(95 \% \mathrm{Cl})\end{array}$ & & $\begin{array}{c}\text { Univariate HR } \\
(95 \% \mathrm{Cl})\end{array}$ & $\begin{array}{c}\text { Multivariate HR } \\
(95 \% \mathrm{Cl})\end{array}$ \\
\hline Older age & $\mathbf{1 . 0 7 ( 1 . 0 3 - 1 . 1 3 )}$ & $1.04(1.00-1.10)$ & & $1.00(0.96-1.05)$ & $1.01(0.97-1.06)$ \\
\hline Female sex & $0.32(0.08-1.02)$ & $0.60(0.15-1.91)$ & & $0.65(0.16-2.20)$ & $0.36(0.06-1.48)$ \\
\hline NHND presentation & $\mathbf{3 2 . 9 1 ( 9 . 4 0 - 1 7 3 )}$ & $11.49(3.19-63)$ & & $1.52(0.16-6.89)$ & $1.23(0.12-6.77)$ \\
\hline Hemorrhage presentation & $1.27(0.01-9.93)$ & $3.49(0.02-47)$ & & $\mathbf{2 2 . 5 9 ( 5 . 7 6 - 8 4 )}$ & $17.67(2.99-117)$ \\
\hline Leptomeningeal drainage & $\mathbf{2 3 . 6 6 ( 3 . 1 4 - 3 0 2 9 )}$ & $\mathbf{5 . 0 3 ( 0 . 4 2 - 6 9 4 )}$ & & $\mathbf{1 7 . 6 1 ( 2 . 2 7 - 2 2 6 6 )}$ & $10.39(1.11-1384)$ \\
\hline Venous ectasia & $\mathbf{4 . 2 1 ( 1 . 0 3 - 1 3 )}$ & $1.17(0.28-3.87)$ & & $\mathbf{7 . 3 8 ( 1 . 7 6 - 2 5 . 3 4 )}$ & $1.89(0.36-7.83)$ \\
\hline
\end{tabular}

Boldface type indicates statistical significance.

CI 1.03-13) were significant risk factors for the occurrence of NHND (Table 3). Female sex and hemorrhagic presentation were not significant. On multivariate analysis, NHND presentation (HR 11.49, 95\% CI 3.19-63) and leptomeningeal venous drainage (HR 5.03, 95\% CI 0.42-694) remained significant risk factors for NHND. On univariate analysis, hemorrhagic presentation (HR 22.59, 95\% CI 5.76-84), leptomeningeal venous drainage (HR 17.61, 95\% CI 2.27-2266), and venous ectasia (HR 7.38, 95\% CI 1.76-25.34) were significant risk factors for hemorrhage, whereas older age, female sex, and NHND presentation were not. Hemorrhagic presentation (HR 17.67, 95\% CI 2.99-117) and leptomeningeal venous drainage (HR 10.39, 95\% CI 1.11-1384) remained significant risk factors for hemorrhage on multivariate analysis (Fig. 2).

\section{Discussion}

Houser et al. were the first to publish data on the importance of the presence or absence of "pial" venous drainage as a determinant for risk of hemorrhage from cerebral dAVFs. ${ }^{7}$ Referred to most frequently as "cortical venous drainage" in the literature, $, 3,14,15$ the more broad term "leptomeningeal venous drainage" more clearly refers to venous outflow from a dAVF that can be associated with a risk of venous hypertension and resultant NHND or hemorrhage. A dichotomous classification that stratifies dAVFs into low risk (those without leptomeningeal venous drainage and thus without risk of NHND or hemorrhage) and high risk (those with leptomeningeal venous drainage) is a simple, reliable scheme for patient counseling that has withstood the test of time and has been supported by multiple studies. ${ }^{1,3,6,7,12-15}$

Soon after the report by Houser et al., Djindjian and Merland delineated 4 types of dAVFs. ${ }^{4}$ Type I dAVFs drain into a venous sinus, Type II dAVFs drain into a venous sinus with leptomeningeal venous reflux, Type III dAVFs drain directly into leptomeningeal veins, and Type IV dAVFs drain directly into ectatic leptomeningeal veins. Decades later, Borden et al. used the cerebral dAVF Djindjian Type I-III designations to describe spinal arteriovenous shunts. ${ }^{2}$ While few use the Borden-Schucart scheme for spinal arteriovenous shunts, the modern literature often cites a Type I-III classification scheme for cerebral dAVFs. ${ }^{3,13-15}$ Given recent data demonstrating the importance of venous ectasia as a potential risk fac- tor for hemorrhage, ${ }^{3,6}$ it would seem prudent to reinstate the Djindjian Type IV as a separate subgroup connoting the highest-risk venous drainage pattern for dAVFs. Our results demonstrated a fairly similar annualized rate of NHND for Type II-IV dAVFs (range 4.5\%-6.0\% per year) and a trend toward an increasing annual rate of hemorrhage for each type: $3.4 \%$ per year for Type II, $4.0 \%$ per year for Type III, and $9.1 \%$ per year for Type IV. Venous ectasia was a significant risk factor for hemorrhage on univariate analysis and demonstrated a trend toward significance on multivariate analysis that was likely limited by our small sample of Type IV dAVFs (29 lesions).

Our results demonstrated that low-risk/Type I dAVFs have a benign natural history from the perspective of NHND and hemorrhage risk. It is imperative to evaluate the natural history of such lesions exclusive of partially treated lesions. Partial treatment can occlude benign venous outflow pathways and incite outflow into leptomeningeal veins. While Satomi et al. cited a $2 \%$ risk for the development of leptomeningeal venous drainage, this risk is difficult to precisely quantify. ${ }^{11}$ Low-risk dAVFs can spontaneously thrombose. In addition, it is difficult to rationalize performing empirical angiographic follow-up in patients with low-risk dAVFs being managed conservatively. Results of angiographic follow-up of these lowrisk fistulas would probably be biased by those causing worsening symptoms or those associated with subsequent hemorrhage due to the development of leptomeningeal venous drainage. Nevertheless, it is clear from our results and those of Satomi et al. that these are relatively benign dAVFs from the perspective of NHND and hemorrhage risk.

Consistent with prior reports, higher-risk (Type II-IV) dAVFs were generally found in older patients and in males, the latter in particular for Type IV dAVFs (72\% males). ${ }^{3,6,13}$ Our results reinforced the importance of leptomeningeal venous drainage as a significant risk factor for NHND and hemorrhage. Importantly, NHND presentation was an independent risk factor for subsequent NHND, and hemorrhagic presentation was an independent risk factor for subsequent hemorrhage. Patients with Type II-IV dAVFs that had not presented with NHND or hemorrhage had annual NHND and hemorrhage rates of $2.3 \%$ and $2.9 \%$, respectively. Those presenting with NHND had a subsequent annual risk of $23.1 \%$ for NHND, whereas those presenting 

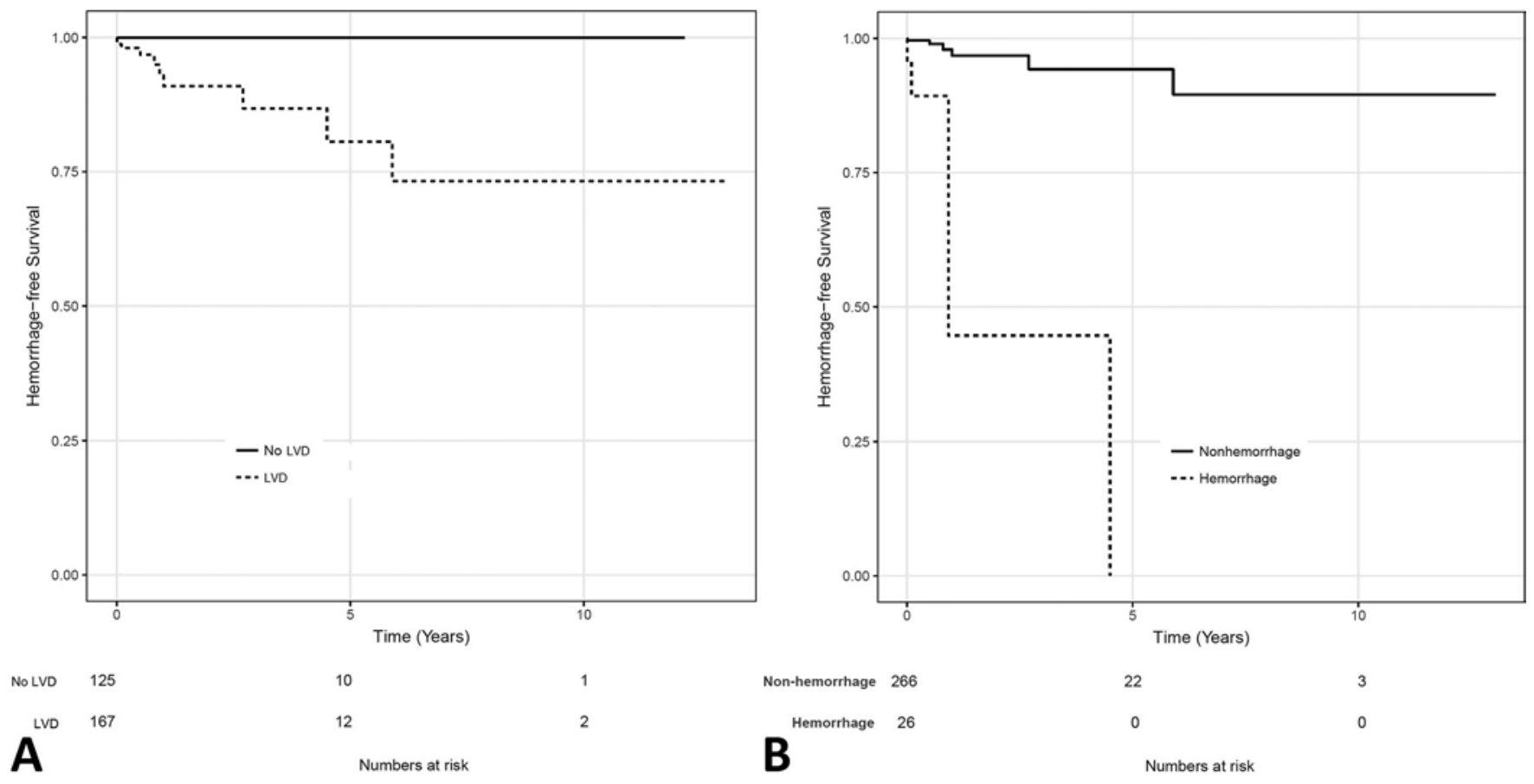

FIG. 2. Kaplan-Meier curves for hemorrhage-free survival comparing dAVFs with and without leptomeningeal venous drainage (LVD) (A) and comparing dAVFs presenting with hemorrhage and those presenting without hemorrhage (B).

with hemorrhage had a subsequent annual rebleeding rate of $46.2 \%$. Although the latter rate is derived from a patient cohort with a shorter mean follow-up period, even if the rehemorrhage rate attenuated over a longer follow-up period, this compelling early rehemorrhage rate mandates treatment in this cohort of patients.

In our prior pooled analysis of the literature, we found a similar annual rehemorrhage rate. ${ }^{6}$ Rates of NHND and hemorrhage for dAVFs that had not presented with NHND or hemorrhage were $0 \%$ and $2 \%$, respectively. It is possible that the substantial proportion of patients with partially treated lesions that was incorporated in our pooled analysis derived some protection from the treatment, highlighting the importance of the current analysis that exclusively evaluates untreated dAVFs. With individualized data, we were able to perform survival analysis to clearly illustrate the independent importance of NHND presentation in relation to the risk of subsequent NHND and hemorrhagic presentation in relation to the risk of subsequent hemorrhage.

Given our study results, all dAVFs with leptomeningeal venous drainage should be considered for treatment. Even dAVFs that do not present in association with NHND or hemorrhage still have a compounded annual risk of 5.2\% for NHND or hemorrhage - a relatively high event rate as compared with the rates for most vascular malformations of the central nervous system. ${ }^{5}$ Our results also encourage relatively expedient treatment of dAVFs presenting with associated NHND or hemorrhage or with venous ectasia. Therefore, our ability to better understand the natural history of symptomatic dAVFs and Type IV dAVFs is likely to remain relatively limited. Note that $129 \mathrm{dAVFs}$ were excluded from the current analysis because of the initiation of treatment prior to a 1-month follow-up period, which excluded a large proportion of Type IV, ethmoidal, and symptomatic dAVFs. Furthermore, the Type IV and hemorrhagic dAVFs included in this study had relatively short follow-up periods. This inherent selection bias comes out of intuitive practicality from 3 institutions equipped to treat these dAVFs upon referral. All patients surviving NHND or hemorrhage from a dAVF were ultimately treated. While a study evaluating a more longitudinal natural history of Type IV and hemorrhagic dAVFs may be of academic value, it would have poor internal and external validity to guide modern practice.

\section{Conclusions}

This multi-institutional analysis of the untreated course of dAVF demonstrated leptomeningeal venous drainage and presentation modality to be significant risk factors for subsequent hemorrhage. Venous ectasia is also a likely risk factor for hemorrhage; a strong trend was seen in our analysis and probably did not meet statistical significance because of inadequate statistical power. All dAVFs with leptomeningeal venous drainage should be considered for treatment given their aggressive natural history; those with venous ectasia or presenting with NHND or hemorrhage should be treated as soon as feasible.

\section{References}

1. Awad IA, Little JR, Akarawi WP, Ahl J: Intracranial dural arteriovenous malformations: factors predisposing to an aggressive neurological course. J Neurosurg 72:839-850, 1990

2. Borden JA, Wu JK, Shucart WA: A proposed classification 
for spinal and cranial dural arteriovenous fistulous malformations and implications for treatment. J Neurosurg 82:166179, 1995

3. Bulters DO, Mathad N, Culliford D, Millar J, Sparrow OC: The natural history of cranial dural arteriovenous fistulae with cortical venous reflux - the significance of venous ectasia. Neurosurgery 70:312-319, 2012

4. Djindjian R, Merland JJ: Super-Selective Arteriography of the External Carotid Artery. Berlin: Springer, 1978

5. Gross BA, Du R: Diagnosis and treatment of vascular malformations of the brain. Curr Treat Options Neurol 16:279, 2014

6. Gross BA, Du R: The natural history of cerebral dural arteriovenous fistulae. Neurosurgery 71:594-603, 2012

7. Houser OW, Baker HL Jr, Rhoton AL Jr, Okazaki H: Intracranial dural arteriovenous malformations. Radiology 105:55-64, 1972

8. Hurst RW, Bagley LJ, Galetta S, Glosser G, Lieberman AP, Trojanowski J, et al: Dementia resulting from dural arteriovenous fistulas: the pathologic findings of venous hypertensive encephalopathy. AJNR Am J Neuroradiol 19:1267-1273, 1998

9. Lasjaunias P, Chiu M, Ter Brugge K, Tolia A, Hurth M, Bernstein M: Neurological manifestations of intracranial dural arteriovenous malformations. J Neurosurg 64:724-730, 1986

10. Lee PH, Lee JS, Shin DH, Kim BM, Huh K: Parkinsonism as an initial manifestation of dural arteriovenous fistula. Eur J Neurol 12:403-406, 2005

11. Satomi J, van Dijk JM, TerBrugge KG, Willinsky RA, Wallace MC: Benign cranial dural arteriovenous fistulas: outcome of conservative management based on the natural history of the lesion. J Neurosurg 97:767-770, 2002

12. Shin NY, Kwon YS, Ha SY, Kim BM, Kim DI, Kim DJ: Venous angioarchitectural features of intracranial dural arteriovenous shunt and its relation to the clinical course. Neuroradiology 55:1119-1127, 2013
13. Söderman M, Pavic L, Edner G, Holmin S, Andersson T: Natural history of dural arteriovenous shunts. Stroke 39:1735-1739, 2008

14. Strom RG, Botros JA, Refai D, Moran CJ, Cross DT III, Chicoine MR, et al: Cranial dural arteriovenous fistulae: asymptomatic cortical venous drainage portends less aggressive clinical course. Neurosurgery 64:241-248, 2009

15. van Dijk JM, terBrugge KG, Willinsky RA, Wallace MC: Clinical course of cranial dural arteriovenous fistulas with long-term persistent cortical venous reflux. Stroke 33:12331236,2002

\section{Disclosures}

Dr. Jovin has received a grant, nonfinancial, from Fundació Ictus Malaltia Vascular and modest honoraria from Silk Road (consultant); Medtronic and Stryker Neurovascular (consultant/advisory board); and Johnson \& Johnson and Neuravi (consultant). Dr. McDougall has been a consultant for Medtronic, Microvention, and Asahi Tecc.

\section{Author Contributions}

Conception and design: Gross, Du. Acquisition of data: Gross, Albuquerque, McDougall, Jankowitz, Du. Analysis and interpretation of data: Gross, Du. Drafting the article: Gross. Critically revising the article: all authors. Reviewed submitted version of manuscript: all authors. Approved the final version of the manuscript on behalf of all authors: Gross. Administrative/technical/ material support: Gross, Albuquerque, McDougall, Jovin, Du. Study supervision: Gross.

\section{Correspondence}

Bradley Gross, Department of Neurological Surgery, UPMC, Ste. B-400, 200 Lothrop St., Pittsburgh, PA 15213. email: grossb2@ upmc.edu. 